\title{
Factors Influencing the Early Phases of Growth of Aerobacter aerogenes
}

\author{
By S. DAGLEY, E. A. DAWES ANd G. A. MORRISON \\ Department of Biochemistry, School of Medicine, Leeds
}

SUMMARY: The method used in maintaining cultures of Aerobacter aerogenes affects the relationship between duration of lag phase and age of inoculum in a glucose ammonium salt medium. Organisms kept in broth may show pronounced early lag whilst those from agar slopes do not. Previous subcultivation with aeration decreases the lag. Bacteria showing no lag may do so when they have been washed; glutamate, $\alpha$-ketoglutarate and succinate partially remove the lag so produced, whilst malate, fumarate and aspartate do not.

The initial growth rate of light inocula showing no lag is increased by the addition of glutamate, aspartate, $\alpha$-ketoglutarate, succinate and oxalacetate; but pyruvate, fumarate and malate have no action or are slightly inhibitory. The effect of these compounds over a concentration range of 0 to $1.6 \times 10^{-3} \mathrm{~g}$. mol./. was studied quantitatively. Filtrates from growing cultures have a similar effect on initial growth rate; those taken during the late logarithmic phase are more effective than those taken earlier. The effects of both filtrates and added compounds are confined to initial growth; the rate of growth in the later phase, when turbidity becomes visible, remains unaltered. This behaviour is dependent on the amount of $\mathrm{O}_{2}$ available in the medium.

Early lag is a phase of greatly reduced rate of growth rather than complete absence of cell division. Glutamate and other effective compounds increase the rate to values approaching normal.

Lodge \& Hinshelwood (1943b) showed that when a glucose ammonium salt medium was inoculated with bacteria grown in a similar medium, the lag period which developed depended on the 'age' of the inoculating cells. When the latter were transferred in the early logarithmic phase there was a considerable lag ('early lag') which was attributed to the diffusion from the cells of metabolic intermediates necessary to allow rapid cell division to begin. Sterile filtrates from fully grown cultures were able, for example, to remove this lag because they supplied the cells with relatively high concentrations of these postulated intermediates. Kinetic studies of bacterial growth provide a method of investigating the reactions which occur before cell division begins. When growth responses similar to those obtained by filtrate additions are produced by known compounds, information may be furnished about the nature of the metabolites normally present during the early growth phases. When a culture ages it shows 'late lag' when inoculated into fresh medium. Morrison \& Hinshelwood (1949) showed with Escherichia coli that such lag was partially abolished by additions of glutamate, aspartate and other compounds. Whereas diffusion of growth intermediates from the cells may account for early lag, several additional factors undoubtedly determine late lag (Lodge \& Hinshelwood, $1943 \mathrm{~b}$ ). Our interest has been the possibility of studying growth intermediates, and we have investigated the early phases of growth.

The work of Lwoff \& Monod (1947) with Esch. coli and of Ajl \& Werkman 
(1948) with Esch. coli and Aerobacter aerogenes, concerning compounds which can partially reverse the retardation of growth due to $\mathrm{CO}_{2}$ deficiency, has also established the importance of glutamic and aspartic acids and closely related non-nitrogenous compounds in the metabolism of these organisms. It was of interest to study the response to these and other compounds when the carbon dioxide tension was adequate for normal growth. It has already been reported (Dagley, Dawes \& Morrison, 1949a) that under these conditions the lag period produced by the addition of phenol to the culture is largely removed by glutamic and succinic acids.

A study of the literature referred to above reveals that early lag was not observed for inocula of the size (or 'age') for which lag would be expected from the work of Lodge \& Hinshelwood (1948b), although Lwoff \& Monod (1947) found that cells which would grow without lag in test-tubes exhibited prolonged lag in flasks aerated by agitation at normal $\mathrm{CO}_{2}$ tensions. We have observed large differences in lag, with different strains of the same organism but the same size of inoculum, and we have, therefore, studied the influence on lag of various conditions of culture before and during growth in the medium under investigation.

\section{EXPERIMENTAL METHODS}

The organism used throughout this work was Aerobacter aerogenes, N.C.T.C. 418. It was maintained by monthly serial subculture in two ways: (1) on nutrient agar, and (2) in nutrient broth. Growth curves were obtained as follows. Samples of the growing culture $(c .2 \mathrm{ml}$.) were withdrawn by Pasteur pipette at various times and killed by the addition of 2 drops of formalin. The bacterial population was determined turbidimetrically using microcells in a Spekker photoelectric absorptiometer with Ilford filters (neutral H 508 and blue no. 6). A relation between turbidity and total count (haemocytometer) was established. When details of the early stages of growth were required before the culture was turbid, viable counts were made by plating out $0.1 \mathrm{ml}$. of a suitable dilution on nutrient agar. Cultures were grown in Pyrex boiling tubes ( 6 in. $\times \frac{5}{8}$ in.), carefully cleaned by boiling with nitric acid followed by washing with glass-distilled water. Cultures were incubated at $39 \pm 0 \cdot 1^{\circ}$. The adverse effect of traces of heavy metals and other impurities on bacterial growth in simple chemically defined media has been stressed by Poole \& Hinshelwood (1940), Lodge \& Hinshelwood (1939) and by Monod (1942). Reproducibility in quantitative experiments is impossible without these special precautions.

The basal medium contained: $5 \cdot 4 \mathrm{~g} . \mathrm{KH}_{2} \mathrm{PO}_{4} ; 1 \cdot 2 \mathrm{~g} .\left(\mathrm{NH}_{4}\right)_{2} \mathrm{SO}_{4} ; 12 \mathrm{~g}$. glucose and $0 \cdot 4 \mathrm{~g}$. $\mathrm{MgSO}_{4} \cdot 7 \mathrm{H}_{2} \mathrm{O} / \mathrm{l}$. of glass-distilled water, $\mathrm{pH}$ adjusted to $7 \cdot 1$. The salt solution and the glucose solution $(3 \% \mathrm{w} / \mathrm{v})$ were sterilized separately and mixed on cooling. Portions of medium $(25 \mathrm{ml}$.) were dispensed aseptically into sterile boiling tubes. Bacteria for any series of experiments were prepared by three serial subcultures in the basal medium and $0.1 \mathrm{ml}$. of the third culture at the phase of growth under investigation was used as inoculum. Thus the number of cells inoculated, as well as their age, varied. 


\section{Measurement of lag}

For an actively-dividing culture the plot of the logarithm of bacterial population against time is linear and if there is no lag the growth curve will extrapolate to the logarithm of cells inoculated at zero time. If a lag takes place it may be defined as the intercept on a line drawn through the latter value parallel to the time axis. It is still convenient to use this definition even in cases when the transition from lag phase to logarithmic phase is not sharp (Hinshelwood, 1946). In several of our experiments, however, it was not necessary to resort to extrapolation since the lag period was followed directly by viable counts.

To ensure reproducibility of results when the effects of a series of factors affecting growth were compared, media were always inoculated from the same culture at the same time and with the same pipette; controls were run at least in duplicate. Agreement was not considered satisfactory if the times for the controls to reach a certain turbidity did not agree to within $5 \mathrm{~min}$.

\section{RESULTS}

\section{Factors influencing relationship between lag and age of inocula}

The age of a growing culture has been defined arbitrarily as the time taken to reach its present population, starting from a haemocytometer count of unity $\left(1.25 \times 10^{-8}\right.$ cells $/ \mathrm{ml}$; Lodge \& Hinshelwood, 1943b). This age refers to the parent culture and the initial population of a daughter culture inoculated from it was 1/250 of the population of the parent, at that age, in our experiments. The present work was undertaken because we found that inocula derived from a strain maintained on nutrient agar slopes did not show lags of the duration expected from the relationship between lag and age so defined, which Lodge \& Hinshelwood (1943b) established for another strain of Aerobacter aerogenes. There were, however, differences in technique since these workers maintained the organism by monthly subculture in broth, and carried out their experiments in media through which a gentle stream of sterile air was passed. Accordingly, we maintained our strain in this way as well as on slopes and constructed lag-age curves for the strains kept in both ways. Whichever strain was studied it was previously subcultured serially in the basal medium three times before inoculation. The results are summarized in Fig. 1; in unaerated cultures the strain maintained on slopes showed no lag over the range of age investigated (curve $D$ ), whilst that maintained in broth (curve $A$ ) showed pronounced early lag with a minimum in the curve about one-third of the way through the logarithmic phase. For curve $A$ the maximum lag at $350 \mathrm{~min}$. occurred towards the end of the logarithmic phase and after this point, when the cells were no longer dividing, the lag fell sharply. Fig. 1 was constructed by following the growth curves of the daughter cultures turbidimetrically, but the sharp rise in lag at ages of inoculum between 200 and $300 \mathrm{~min}$. in curve $A$ was also confirmed by viable counts for an inoculum taken at $220 \mathrm{~min}$.; it was found that a lag of about $\mathbf{2 4 0} \mathrm{min}$. occurred which was largely removed by addition of glutamate. 
Curves $B$ and $C$ were both obtained with the organism stored in broth and in the preliminary three passages through basal medium the cultures were aerated at normal $\mathrm{CO}_{2}$ tension in the manner adopted by Lodge \& Hinshelwood (1943b). In case $B$, such cells were tested for lag in an aerated medium and, in case $C$, in an unaerated medium. When cells to be grown in conditions of full aeration are not previously trained to these conditions a considerable lag develops.

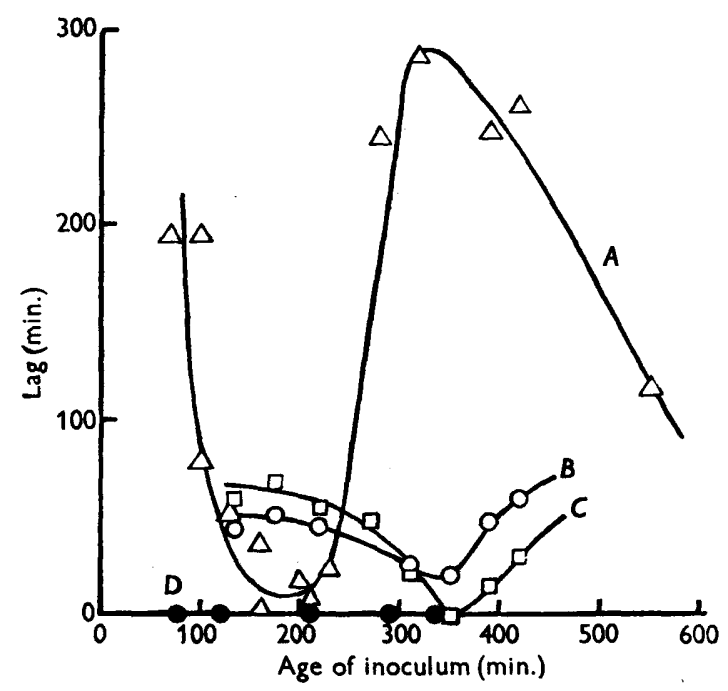

Fig. 1. Effect of method of maintenance of strain on lag-age relationship. Strain maintained: $(A)$ in broth $\triangle$, inocula grown without aeration, tested without aeration; $(B)$ in broth $O$, inocula grown with aeration, tested with aeration; $(C)$ in broth $\square$, inocula grown with aeration, tested without aeration; $(D)$ on slopes $O$, inocula grown without aeration; tested without aeration.

Lwoff \& Monod (1947) observed that cells inoculated into media aerated by agitation in a flask showed lag, and for this reason they added $\mathrm{Na}_{2} \mathrm{~S}$ which acted as a 'facteur de départ' and removed the lag. In our experiments, however, we found that once the bacteria had overcome this lag period in an aerated medium, and were in the logarithmic phase, they were able to grow without abnormal lag in a new aerated medium containing no additional substance. A comparison of curves $A$ and $C$ shows that the cells which were not trained to aeration were also distinguished from those which were so trained by their behaviour in unaerated media.

\section{Effect of various compounds on early phases of growth}

Using inocula of the organism maintained on slopes to give an initial population of $c .2 \times 10^{4}$ bacteria $/ \mathrm{ml}$., it was found that the addition of small amounts of certain compounds enabled the cultures to become turbid before the control did, although in the region of turbidity for which growth curves could be accurately constructed, the growth rates were identical (Fig. 2). The compounds added must have exerted their effect before the cultures became visibly 
turbid. As previously indicated, bacteria stored on agar slopes did not give measurable early lag with this size of inoculum. Viable counts of two cultures, one containing no $\mathrm{Na}$ L-glutamate and the other $6 \times 10^{-3} \mathrm{~g}$. mol./l., showed that the growth rate for the first few generations was markedly increased by the

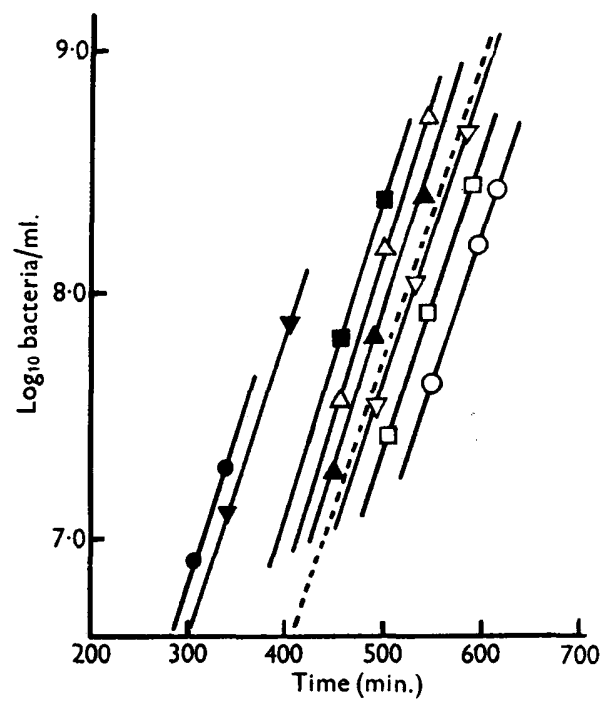

Fig. 2. Sections of growth curves determined by turbidimetric method. Cultures contained various organic acids at 0.06 g./1. Control - --- ; acids : L-glutamic, $\bigcirc$; DL-aspartic, $\nabla$; succinic, $\square$; oxalacetic, $\Delta$; $a$-ketoglutaric, $\Delta$; fumaric, $\nabla ;$ pyruvic, $\square$; malic, 0 .

glutamate, but its effect persisted only to populations which were just visible, i.e. c. $5 \times 10^{6}$ cells $/ \mathrm{ml}$. Further, the growth curve for the control in Fig. 2 extrapolates to the initial population at zero time, so that in the first few generations growth in media containing glutamate, etc., must have been faster than in the control.

The concentration of all the compounds used in the experiments of Fig. 2 was $0.06 \mathrm{~g}$./l. Similar sets of growth curves were obtained at five other concentrations using the same inoculum at the same time. From the six sets of results for each compound, the time displacement of each growth curve relative to that of the control was obtained (Fig. 3). Malate, fumarate and pyruvate were somewhat inhibitory in the initial stages of growth, whereas glutamate, aspartate, succinate and $\alpha$-ketoglutarate promoted growth. The sample of oxalacetate was that used by Morrison \& Hinshelwood (1949), prepared by the method of Fenton \& Jones (1900) and the anomalous shape of the curve for this substance is accounted for by the presence of unoxidized malic acid which is inhibitory.

\section{Lag shown by washed cells}

Although the organism maintained on slopes showed no lag at ages at which other strains did so, lag was obtained by harvesting cells from a culture in basal medium and washing three times with phosphate buffer pH 7.1. Media 
containing glutamate, succinate or $\alpha$-ketoglutarate, when inoculated with cells so treated, became turbid much sooner than the control, but not when aspartate, citrate, acetate, fumarate or malate was added. As with Fig. 2, the growth curves were parallel in the region of visible turbidity, but unlike Fig. 2 extrapolation showed that the control culture had a lag period which was

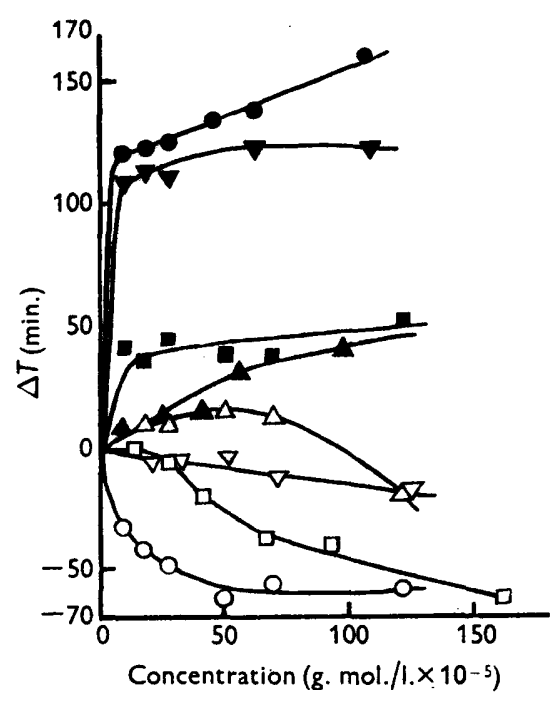

Fig. 3. Effect of concentration of various organic acids on initial growth. $\Delta T$ : displacement of growth curve (min.) with respect to control, positive values denote acceleration and negative values retardation of initial growth. Acids: L-glutamic, $\%$; DL-aspartic, $\nabla$; succinic, $\square$; $\alpha$-ketoglutaric, $\Delta$; oxalacetic, $\triangle$; fumaric, $\nabla$; pyruvic, $\square$; malic, $O$.

decreased by adding glutamate, etc. These results were confirmed by viable counts. The effects produced by various added compounds on the lag shown by washed cells were similar to those described in the previous section, except that aspartate was effective in displacing the growth curve of unwashed cells but not in reducing the lag of washed cells.

\section{The early lag phase}

During the course of this work we obtained through the courtesy of Prof. Sir Cyril Hinshelwood a strain of $A$. aerogenes which, unlike the organism stored on agar slopes which we were using at the time, showed early lag but of much shorter duration than that with strain $A$, Fig. 1, later investigated. However, with small inocula giving an initial population of $300-400$ bacteria/ml., cultures showed lags of $7 \mathrm{hr}$. or more. Growth over this period was followed by plate counts and the effect of additions of glutamate, succinate and $\alpha$-ketoglutarate studied (Fig. 4). Growth rates were also followed at a later stage by turbidity measurements and were in each case the same as the control, although cultures with the additions present reached visible turbidity considerably earlier. Early lag is not a phase where cell division is totally suppressed, but one of greatly reduced growth rate, and the effect of additions of growth- 
promoting substances is to increase the slower growth rate to a value approaching normal. This was eonfirmed in several other experiments using the viable count technique.

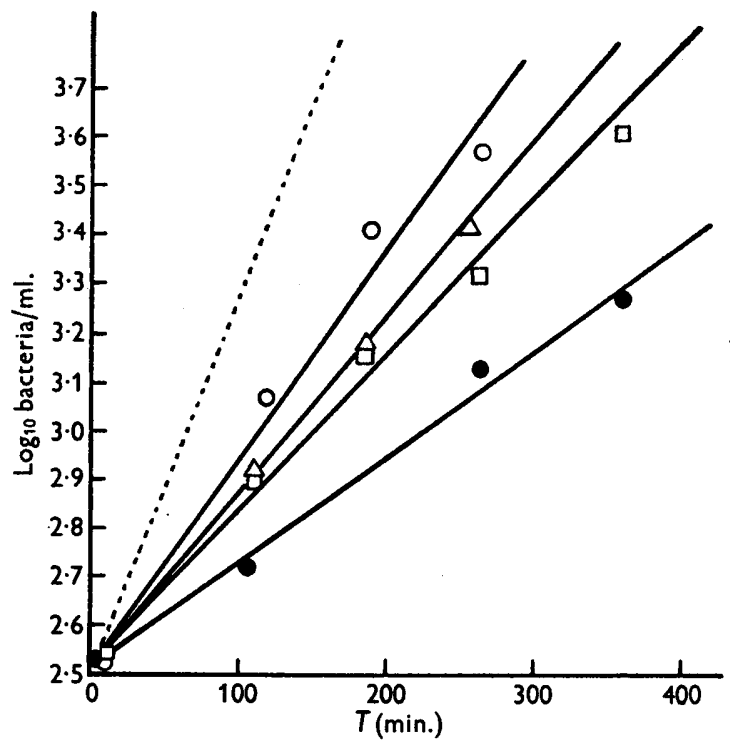

Fig. 4. Early lag of light inocula; effect of organic acids on decreased growth rate as followed by viable counts. Control, 0 . Additions of $3 \times 10^{-4} \mathrm{~g} \cdot \mathrm{mol} . / \mathrm{l}$. of following acids: succinic, $\square$; $\alpha$-ketoglutaric, $\Delta$; L-glutamic, $O$. Dotted line indicates growth rate obtained by turbidimetric method when cultures had grown to populations $>10^{7}$ bacteria/ml. The organism used in these experiments was that obtained from Prof. Sir Cyril Hinshelwood.

Effect of inoculum size and culture filtrates on the early growth phase

Lodge \& Hinshelwood (1943b) showed that sterile filtrates from fully-grown cultures abolished early lag. We observed that media to which such filtrates were added always reached visible turbidity before those with no additions, when a light inoculum was used. By the methods previously described this was shown to be due to the attainment of a higher initial growth rate in the former cases.

Inocula were taken from a growing culture at various times; samples of the same culture were also centrifuged, the clear supernatant boiled and $10 \mathrm{ml}$. additions made to $10 \mathrm{ml}$. lots of basal medium in which the inocula were to be tested. In this way it was possible to study not only the effect of filtrates of different ages on the initial mean generation time but also how the response obtained with various inocula depended on their age and size.

The results of such experiments (Fig. 5) show that (1) filtrates from the late logarithmic phase are more effective than those taken earlier in growth; (2) the response decreases with the age, i.e. with the size of the inoculum. It was also found that the response to glutamate and other compounds depended in a similar way on inoculum size. Although exact extrapolation of the curves in 
Fig. 5 to the point $\Delta T=0$ is difficult, it is possible to estimate that this occurs for ages between 410 and $450 \mathrm{~min}$. Calculation shows that this corresponds to a parent $\log$ (cell population) value of $9 \cdot 1$ to $9 \cdot 4$, which in turn shows that filtrates will have no effect $(\Delta T=0)$, using this technique, when log (initial population) for the daughter culture is $6 \cdot 7-7 \cdot 0$, i.e. when the initial population is 5 to $10 \times 10^{6}$ cells $/ \mathrm{ml}$. This is the point when the turbidity of the culture is sufficient to be measured by our methods, which is in accordance with our observations that filtrates and the various growth-promoting compounds exerted their effects earlier than this point, leaving the growth rate obtained from turbidities unaffected.

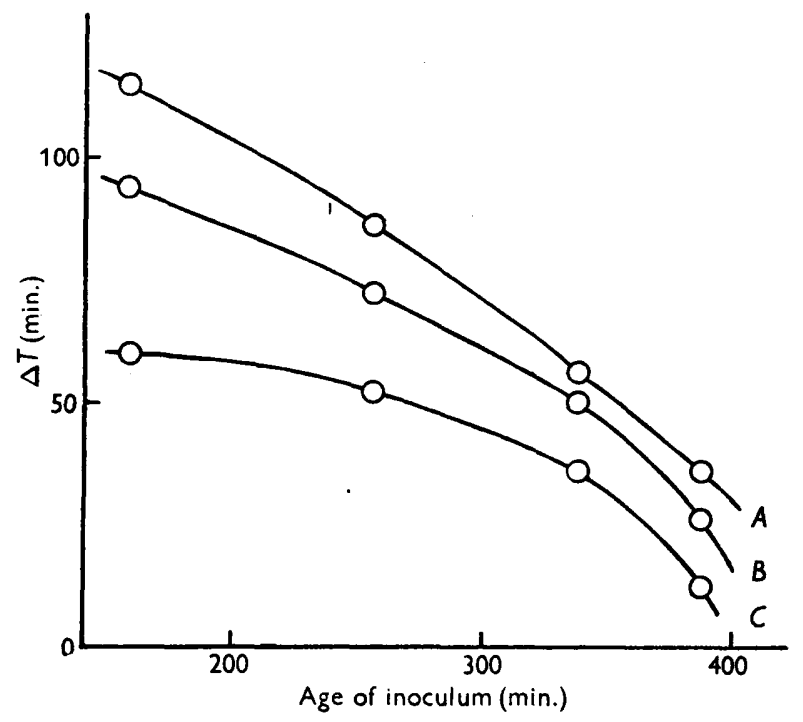

Fig. 5. Effect of culture filtrates and of inoculum size on initial growth. $\Delta T$ : displacement of growth curves with respect to control containing no filtrate addition. $10 \mathrm{ml}$. filtrate, $10 \mathrm{ml}$. basal medium. Filtrates from cultures of ages $(A) 370 \mathrm{~min}$., $(B) 225 \mathrm{~min}$., (C) $125 \mathrm{~min}$.

It was observed by Lodge \& Hinshelwood $(1943 a)$ that when glutamic acid and other amino-acids were the sole source of nitrogen, the culture population did not attain its maximum unless the media were aerated, although the initial growth rate might be greater than in unaerated glucose ammonium salt media. We found that in unaerated cultures with glutamate, succinate or $\alpha$-ketoglutarate as the sole carbon sources, visible turbidity was reached more rapidly than with cultures containing molecular equivalents of glucose; but the rate of growth soon decreased in the former cases, and the final stationary populations were considerably less than with glucose, with which growth also differed in being logarithmic throughout. These observations, together with those previously recorded, suggest that the various factors promoting growth do not exert their maximum effects unless the $\mathrm{O}_{2}$ tension is optimal. We found that when growing cultures contained $0.6 \mathrm{mg}$. methylene blue/l. no decolorizing of 
the dye occurred until visible turbidity was reached, but after this point the rate of reduction was relatively rapid so that a culture of $8 \times 10^{7} \mathrm{cells} / \mathrm{ml}$. decolorized the dye in less than $30 \mathrm{~min}$. and $3 \times 10^{8}$ cells $/ \mathrm{ml}$. did so in $15 \mathrm{~min}$. Colour returned when cell division ceased in the stationary phase. Two salient facts about the mid-logarithmic phase of cells grown under these conditions, therefore, emerge: (1) the bacteria deplete the medium of $\mathrm{O}_{2}$; (2) experiments with filtrates prove that compounds are present which can stimulate faster growth when transferred to a medium not depleted of $\mathrm{O}_{2}$. Accordingly, we studied the growth of two cultures, both in basal medium containing no additions but one culture was grown in an unaerated tube, while through the other a stream of air of normal $\mathrm{CO}_{2}$ content was passed as soon as visible turbidity was reached. In the latter culture there was a small increase in growth rate from the moment aeration began, and growth continued at this rate to give a stationary population higher than in the unaerated culture. Lodge \& Hinshelwood (1943a) found a mean generation time of $\mathbf{3 2} \mathrm{min}$. for $A$. aerogenes when fully aerated, whilst when unaerated it was $40 \mathrm{~min}$.

\section{DISCUSSION}

In our experiments previous subcultivation ensured that over thirty generations of bacteria had been produced when growing with glucose as sole carbon source (apart from $\mathrm{CO}_{2}$ ) prior to inoculation into the experimental media. The fact that glutamic and aspartic acids, and non-nitrogenous compounds related to them, were able in small amounts to accelerate the initial processes of growth in the basal medium, whether by abolishing lag or increasing growth rate, suggests that they take part in rate-controlling reactions. This is supported by the observation that filtrates from growing cultures produced responses similar to the compounds tested, under the same conditions. These conclusions accord with those of Morrison \& Hinshelwood (1949), who studied the effects produced by various compounds on the lag which results when cells are allowed to age. They also obtained evidence that the rate-controlling step was the production of the carbon skeletons necessary for synthesis from the glucose, and that subsequent amination was relatively rapid; they assigned to glutamate a key role in terminating lag. Roine (1947), working with a strain of Torulopsis utilis, produced evidence for the view that glutamic acid is the primary aminoacid formed in yeast from a carbon chain and ammonia, and is of first importance in the bio-synthesis of amino-acids and proteins.

Our experiments showing the dependence of early lag on the method of maintaining the cultures whereby the synthetic abilities of the bacteria in a basal glucose ammonium salt medium are undoubtedly modified, are compatible with the view of Lodge \& Hinshelwood (1943b) that the early lag phase is governed by the rate of synthesis of essential metabolites. Our studies of the lag produced by washing are also readily interpreted by assuming that washing removes essential intermediates from the cells, just as high dilution can give rise to early lag by a similar process.

We found that culture filtrates and various active compounds affected initial growth but not growth in the later logarithmic phase, and we suggest that they 
are able to exert their maximum effect only when the $\mathrm{O}_{2}$ tension in the medium is optimal. There is abundant evidence for considerable changes in redox potential during bacterial growth (Hewitt, 1936; O'Meara, McNally \& Nelson, 1947) and optimal requirements may, in consequence, be assumed to vary with the phase in the growth process. Thus, we found that bacteria lagged considerably in fresh medium through which air was passed, although when aerated in mid-logarithmic growth, when experiments with methylene blue indicated oxygen depletion, growth was accelerated. In their experiments on the role of $\mathrm{CO}_{2}$ in bacterial growth, Lwoff \& Monod (1947) also observed the adverse effect of aeration on light inocula. They postulated that reactions concerned with the synthesis or metabolism of glutamic acid are related to a favourable cellular redox potential, the establishment of which is rendered difficult by excessive aeration. This difficulty was not met when they grew their cultures in tubes instead of agitated flasks; but we found (Dagley $e t$ al. $1949 a$ ) that $\mathrm{Na}_{2} \mathrm{~S}$, added to flasks by Lwoff \& Monod (1947) as 'corps réducteur "protecteur", in order to remove lag, also promoted growth in tubes. Winslow, Walker \& Sutermeister (1932) and Lodge \& Hinshelwood (1943 $a$ ) assumed that increased growth in aerated cultures was due to removal of toxic products by the air stream, but from our experiments showing the relationship of aeration to growth response it would appear that $\mathrm{O}_{2}$ has a more positive function and ensures the maximum rate of utilization of essential metabolites by the cells.

Ajl \& Werkman (1948) drew attention to the fact that compounds compensating for $\mathrm{CO}_{2}$ deficiency are also constituents of the Krebs oxidation cycle or are their natural precursors. For several reasons, however, a view assigning to this cycle a significant role in bacterial metabolism must be treated with reserve. As Ajl \& Werkman (1949) pointed out, several of these compounds replace $\mathrm{CO}_{2}$ anaerobically, and it is generally considered that the tricarboxylic acid cycle is an aerobic mechanism by which various substances can be oxidized to $\mathrm{CO}_{2}$ and $\mathrm{H}_{2} \mathrm{O}$ to yield energy to the system. We also found that whereas some compounds in the cycle accelerated growth others did not; it is therefore difficult to see why the latter should be prevented from entering this cycle, if it exists in bacterial systems. Furthermore, we found (Dagley et al. $1949 b$ ) that fluoroacetate, which blocks this cycle (Buffa, Coxon, Liébecq \& Peters, 1949) had no effeet on the growth of $A$. aerogenes over a range of concentration at which iodoacetate was strongly bacteriostatic. It is well established that the action of the latter is entirely different from that of fluoroacetate, since it combines with $-\mathrm{SH}$ groups.

One of us (G.A.M.) wishes to acknowledge the award of an Imperial Chemical Industries Ltd. Research Fellowship.

\section{REFERENCES}

AJL, S. J. \& Werkman, C. H. (1948). Replacement of $\mathrm{CO}_{2}$ in heterotrophic metabolism. Arch. Biochem. 19, 483.

AJl, S. J. \& Werkman, C. H. (1949). Anaerobic replacement of carbon dioxide. Proc. Soc. exp. Biol., N.Y., 70, 522. 
Buffa, P., Coxon, R. V., Likbecq, C. \& Peters, R. A. (1949). Experiments on the tricarboxylic acid cycle in brain and kidney tissues. 1st Internat. Congr. Biochem. Abstr. No. 52/3, p. 570.

Dagley, S., Dawes, E. A. \& Morrison, G. A. (1949a). Application of bacterial kinetics to biochemical problems. Nature, Lond., 163, 532.

Dagley, S., Dawes, E. A. \& Morrison, G. A. (1949b). Influence of amino-acids, and compounds in the Krebs oxidation cycle, on 'early lag'. Biochem. J. 45, xxvi.

Fenton, H. J. H. \& Jones, H. O. (1900). The oxidation of organic acids in presence of ferrous iron. Part I. J. chem. Soc. 77, 69.

HEwITT, L. F. (1936). Oxidation reduction potentials in bacteriology and biochemistry, 4th ed. London: London County Council.

Hinshelwood, C. N. (1946). The chemical kinetics of the bacterial cell. Oxford University Press.

Lodge, R. M. \& Hinshelwood, C. N. (1939). Physicochemical aspects of bacterial growth. Part IV. Conditions determining stationary populations and growth rates of Bact. Lactis Aerogenes in synthetic media. J. chem. Soc. p. 1688.

Lodge, R. M. \& Hinshelwood, C. N. (1943a). Physicochemical aspects of bacterial growth. Part VIII. Growth of Bact. Lactis Aerogenes in media containing ammonium sulphate or various amino acids. J. chem. Soc. p. 208.

Lodge, R. M. \& Hinshelwood, C. N. (1943b). Physicochemical aspects of bacterial growth, Part IX. The lag phase of Bact. Lactis Aerogenes. J. chem. Soc. p. 213.

Lworf, A. \& Monod, J. (1947). Essai d'analyse du role de l'anhydride carbonique dans la croissance microbienne. Ann. Inst. Pasteur, 73, 323.

MonoD, J. (1942). Recherches sur la croissance des cultures bactériennes. Paris: Herman \& Cie.

Morrison, G. A. \& Hinshelwood, C. N. (1949). Nitrogen utilization and growth of coliform bacteria. Part III. Nitrogen utilization and lag phase. J. chem. Soc. p. 380.

O'Meara, R. A. Q., McNally, P. A. \& Nelson, H. G. (1947). The intra-cellular mode of action of the sulphonamide derivatives. Lancet, ii, $7 \mathbf{7 7}$.

Poole, A. \& Hinsherwood, C. N. (1940). Physicochemical aspects of bacterial growth. Part VI. The influence of toxic substances on growth rate, stationary population and fermentation reactions of Bact. Lactis Aerogenes. J. chem. Soc. p. 1565.

RorNe, P. (1947). On the formation of primary amino acids in the protein synthesis in yeast. Ann. Acad. Sci. Fennicae, A, 11, 26.

Winslow, C.-E. A., Walker, H. H. \& Sutrermeister, M. (1932). The influence of aeration and of sodium chloride upon the growth curve of bacteria in various media. J. Bact. 24, 185.

(Received 17 January 1950) 